\title{
CORRELATION OF INFRARED EMISSION WITH H I AND CO GAS IN THE HIGH LATITUDE CLOUD AREA L1642
}

\author{
T. Liljeström \\ Helsinki University Observatory \\ Tähtitorninmäki, \\ SF-00130 Helsinki Finland
}

\author{
R. Laureijs \\ Kapteyn Astronomical Institute and \\ Laboratory for Space Research \\ P.O. Box 800 \\ 9700 AV Groningen The Netherlands
}

The high-galactic-latitude cloud $\mathrm{L} 1642\left(l=210.8^{\circ}, b=-36.7^{\circ}\right)$ is a suitable candidate to relate IR measurements with atomic and molecular data because it has a reasonable size with respect to the rather poor (IRAS) IR resolution, a moderate optical extinction and an isolated location in the direction towards the galactic anticenter. The exceptionally high galactic latitude of $-36.7^{\circ}$ implies that L1642 is some $60 \mathrm{pc}$ below the galactic plane (if $r \approx 100 \mathrm{pc}$ is adopted for its distance). L1642 is thus sufficiently far off the galactic plane to minimize the confusion by background gas and dust clouds.

The large scale structure of the $100 \mu \mathrm{m}$ emission in the L1642 area, presented in Laureijs et al. (1987) and Liljeström and Mattila (1988), shows that the dark cloud L1642 is located in the head of a comet-shaped nebula with a long tail pointing towards the galactic plane. Figures $1 a$ and $b$ show the IRAS surface brightness maps of L1642 at $12 \mu \mathrm{m}$ and $100 \mu \mathrm{m}$ smoothed to a $9^{\prime}$ Gaussian beam. It is seen that the $100 \mu \mathrm{m}$ emission has a maximum in the center of the cloud whereas the brightness distribution at $12 \mu \mathrm{m}$ shows maximum intensities in the outer parts of the cloud. The large scale structure of the integrated $\mathrm{H} \mathrm{I}$ line intensity has a morphology similar to the IRAS $12 \mu \mathrm{m}$ map whereas the integrated CO line emission shows a morphology similar to the IRAS $100 \mu \mathrm{m}$ map.

The relationship I(CO) vs. $A_{\mathrm{V}}$ reveals a linear correlation of $3.3 \mathrm{~K} \mathrm{~km} \mathrm{~s}^{-1} \mathrm{mag}^{-1}$ for

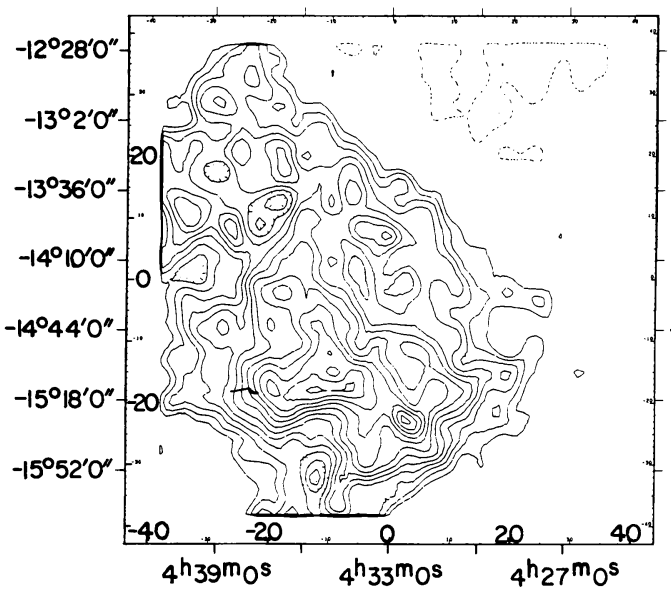

Figure 1a. IRAS surface brightness distributions at $12 \mu \mathrm{m}$. The contour levels are in steps of $0.05 \mathrm{MJy} \mathrm{sr}^{-1}$ with the highest contour at 0.75 MJy $\mathrm{sr}^{-1}$.

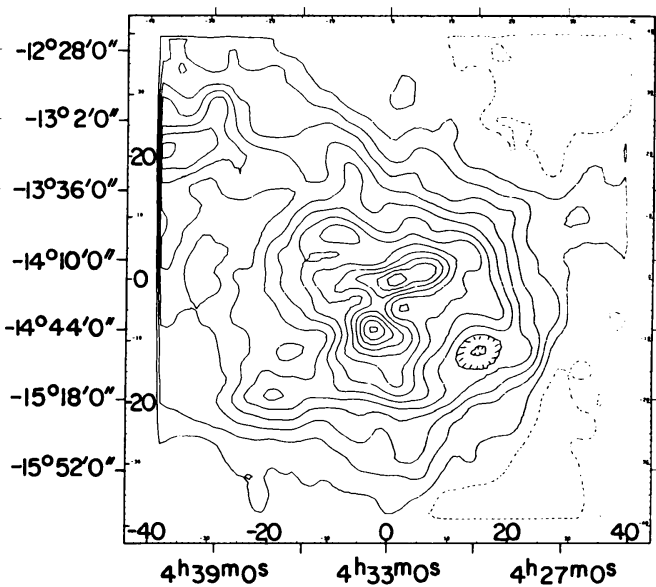

Figure 1b. IRAS surface brightness distribution at $100 \mu \mathrm{m}$. The contour levels are in steps of $1.0 \mathrm{MJy} \mathrm{sr}^{-1}$ with the highest contour at $13 \mathrm{MJy}$ $\mathrm{sr}^{-1}$.

S. Bowyer and C. Leinert (eds.), The Galactic and Extragalactic Background Radiation, 216-217.

(C) 1990 IAU. Printed in the Netherlands. 
$A_{\mathrm{V}}>1.1$ mag. When $A_{\mathrm{V}}<1.1 \mathrm{mag}$ there is a big scatter in the data, which is best understood with the inhomogeneous cloud structure in the outer parts of the molecular cloud where the CO abundance is sensitive to temperature (Langer 1976). Also, the $I(C O)$ vs. $I(100 \mu \mathrm{m})$ relationship shows this scatter, probably due to the same reason as above, or to a dust confusion of the expanding Eridanus H I shell present in the L1642 area (Liljeström and Mattila 1988). If all data points are considered a correlation $\mathrm{I}(\mathrm{CO}) / \mathrm{I}(100 \mu \mathrm{m})=(0.5 \pm 0.1) \mathrm{K} \mathrm{km} \mathrm{s}^{-1} /\left(\mathrm{MJy} \mathrm{sr}^{-1}\right)$ is obtained. However, if only the stronger $100 \mu \mathrm{m}$ emission $\left(>10 \mathrm{MJy} \mathrm{sr}^{-1}\right)$ is considered the $\mathrm{I}(\mathrm{CO})$ vs. $\mathrm{I}(100 \mu \mathrm{m})$ correlation factor is $(0.86 \pm 0.35) \mathrm{K} \mathrm{km} \mathrm{s}^{-1} /\left(\mathrm{MJy} \mathrm{sr}^{-1}\right)$, which agrees with the mean value of $0.9 \mathrm{~K} \mathrm{~km} \mathrm{~s}^{-1} /\left(\mathrm{MJy} \mathrm{sr}^{-1}\right)$ found by Weiland et al. (1986).

The contour map of the $\mathrm{H}$ I line width (Figure 2) in the SE area of the cloud shows an increase from 2.6 to $4.0 \mathrm{~km} \mathrm{~s}^{-1}$. The spectra do not reveal any sign of a second velocity component at the $\mathrm{L} 1642$ cloud velocity $\left(\sim 1 \mathrm{~km} \mathrm{~s}^{-1}\right)$, which could explain the line broadening as a blend effect. Although an explanation in terms of increased turbulence cannot be excluded, the fact that the IRAS $12 \mu \mathrm{m}$ surface brightness map (Figure $1 a$ ) shows the big maximum close to the $\mathrm{H}$ I line broadening maximum supports a thermal line broadening model, where the heating of the $\mathrm{HI}$ gas is due to the photoelectric emission from very small grains and/or PAHs.

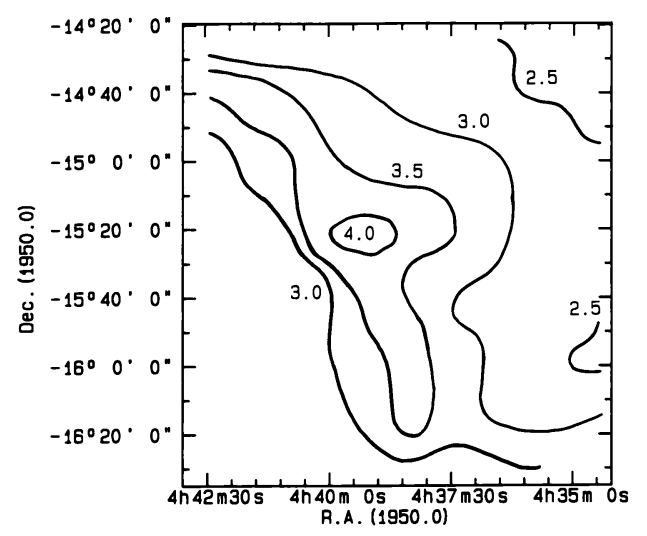

Figure 2. Spatial distribution of the $\mathrm{H}$ I line width in the SE area of the cloud. Unit is $\mathbf{k m}$ $\mathrm{s}^{-1}$.

Assuming that the turbulent and large scale motions are the same in the broadened $\mathrm{H} \mathrm{I}$ line region and immediately outside this area, a temperature enhancement of about $200 \mathrm{~K}$ is obtained for the $\mathrm{H} \mathrm{I}$ gas according to the increase of the line width from 2.6 to $4.0 \mathrm{~km} \mathrm{~s}^{-1}$. Adopting for the $\mathrm{L} 1642 \mathrm{H}$ I cloud the temperature $T_{\mathrm{K}}=81 \mathrm{~K}$, an average value for diffuse clouds (Spitzer and Cochran 1973), the increase of the $\mathrm{H}$ I line width corresponds to an increase of $T_{\mathrm{K}}$ from $\sim 80$ to $280 \mathrm{~K}$. This result indicates that the dark cloud L1642 is embedded in a lukewarm H I envelope. As in the case of L1642, filamentary structures are often associated with these warm $\mathrm{H}$ I envelopes. Additionally, it is known that $\mathrm{H}$ I clouds get warmer at higher $|z|$ (Kulkarni and Heiles 1988).

\section{REFERENCES}

Kulkarni, S.R. and Heiles, C. 1988, in Galactic and Extragalactic Radio Astronomy, eds. G.L. Verschuur and K.I. Kellermann (New York: Springer-Verlag, Inc.), p. 132-133.

Langer, W. 1976, Ap. J., 184, 269.

Laureijs, R., Mattila, K., and Schnur, G. 1987, Astron. Astrophys., 184, 269.

Liljeström, T. and Mattila, K. 1988, Astron. Astrophys., 196, 243.

Spitzer, L., Jr. and Cochran, W. D. 1973, Ap. J. (Letters), 186, L23.

Weiland, J. et al. 1986, Ap. J. (Letters), 306, L101. 